\title{
A Candidate Wide Brown Dwarf Binary in the Argus AssociATION: 2MASS J14504216-7841413 AND 2MASS J14504113-7841383
}

\author{
Adam J. Burgasser, ${ }^{1}$ Dagny L. Looper, ${ }^{2}$ and J. Davy Kirkpatrick ${ }^{3}$ \\ ${ }^{1}$ Center for Astrophysics and Space Science, University \\ of California San Diego, La Jolla, CA 92093, USA \\ ${ }^{2}$ New York University Tisch School of the Arts, 721 \\ Broadway, 10th Floor, New York, NY 10003, USA \\ ${ }^{3}$ IPAC, Mail Code 100-22, Caltech, 1200 E. California Blvd. Pasadena, CA 91125.
}

Keywords: stars: binaries: general — stars: fundamental parameters - stars: individual (2MASS J14504216-7841413, 2MASS J14504113-7841383) — stars: low mass, brown dwarfs 
Widely-separated ( $\gtrsim 100 \mathrm{AU}$ ) multiples are rare among the lowest mass stars and brown dwarfs (Caballero 2007; Kraus \& Hillenbrand 2009), and often (but not exclusively) associated with young ( $\lesssim 100 \mathrm{Myr})$, nearby stellar associations (e.g., Close et al. 2007). We report the discovery of a wide, very low mass, and potentially young binary, 2MASS J14504216-7841413 and 2MASS J14504113-7841383 (hereafter 2MASS J1450-7841AB). The primary was initially identified in the DENIS (Epchtein et al. 1997) and 2MASS (Skrutskie et al. 2006) surveys as a candidate late-M dwarf based on its red optical/infrared colors (DENIS $I=18.03 \pm 0.16, I-J$ $\left.=3.32 \pm 0.19,2 \mathrm{MASS} J=14.71 \pm 0.06, J-K_{s}=1.38 \pm 0.08\right)$. A faint red companion, 2MASS J14504113-7841383, is present in the 2MASS Point Source Catalog $(J=$ $\left.15.64 \pm 0.09, J-K_{s}=1.28 \pm 0.14\right)$ separated by $4^{\prime \prime} .23 \pm 0^{\prime \prime} .11$ at position angle $314^{\circ}$.

We observed 2MASS J1450-7841AB with the Low Dispersion Survey Spectrograph 3 (LDSS-3; Allington-Smith et al. 1994) on the Magellan 6.5m Clay Telescope on 2007 May 9 (UT). The system was resolved in $i$ - and $z$-band imaging data at an identical separation $\left(4^{\prime \prime} .28 \pm 0^{\prime \prime} 14\right.$ at $314^{\circ}$; Figure 1$)$ as $2 \mathrm{MASS}$, with $\Delta i=1.03 \pm 0.05$ and $\Delta z$ $=1.01 \pm 0.05$. Combining astrometry from 2MASS and LDSS-3, we measure identical proper motions of $\left(\mu_{\alpha} \cos \delta, \mu_{\delta}\right)=(22 \pm 32,-59 \pm 34)$ mas/yr for 2MASS J1450-7841A and $(-12 \pm 23,-4 \pm 23) \mathrm{mas} / \mathrm{yr}$ for 2MASS J1450-7841B.

LDSS-3 red optical spectroscopy (Figure 1; cf. Burgasser \& Kirkpatrick 2006) reveals strong $\mathrm{TiO}$ and $\mathrm{VO}$ absorption bands in both sources, typical of late-M dwarfs. Comparing to spectral templates (Bochanski et al. 2007), we estimate types of M8p and M9p for 2MASS J1450-7841A and B, respectively. The "peculiar" designations reflect the strong VO bands and weak $\mathrm{Na}$ I lines, consistent with low surface gravities (Kirkpatrick et al. 2008; Cruz et al. 2009). We detect weak $\mathrm{H} \alpha$ emission from 2MASS J1450-7841B, but the data are inadequate to infer the presence of Li I in either spectrum.

Using the young and field $M_{J}$ /spectral type relations of Faherty et al. (2016), we estimate equivalent distances of $75 \pm 25$ pc for 2MASS J1450-7841A and B, implying a projected separation of $\sim 300$ AU. Applying this distance estimate and the proper motions to the BANYAN II tool (Gagné et al. 2014; Malo et al. 2013), we find a 93\% probability of membership in the $\sim 40$ Myr Argus Association (Zuckerman et al. 2011). At this age, 2MASS J1450-7841A and B have masses of $\sim 0.04 \mathrm{M}_{\odot}$ (Baraffe et al. 2003), well below the hydrogen fusion limit. Confirmation and study of this potentially young and unusually wide substellar binary is warranted.

This paper includes data gathered with the 6.5 meter Magellan Telescopes located at Las Campanas Observatory, Chile. 


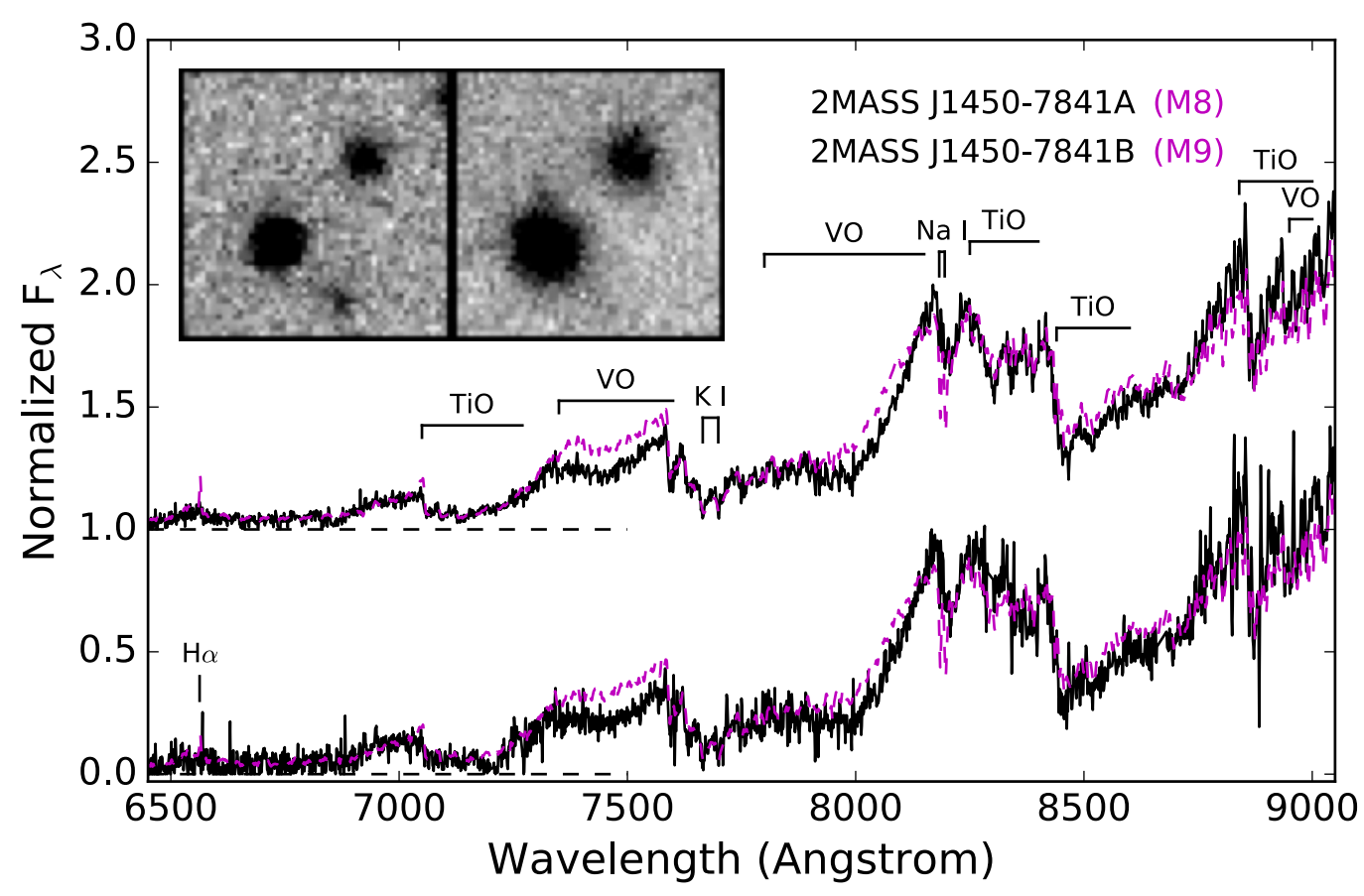

Figure 1. LDSS-3 spectra of 2MASS J1450-7841A (top) and B (bottom), corrected for telluric absorption and normalized at $8170 \AA \AA$; compared to M8 and M9 templates (dashed magenta lines). Key absorption features are labeled. Inset shows LDSS-3 $i$-band (left) and $z$-band (right) images of 2MASS J1450-7841AB, 9".5 on a side and oriented with North up and East to the left. 2MASS J1450-7841A is the southeastern component.

\section{REFERENCES}

Allington-Smith, J., Breare, M., Ellis, R., et al. 1994, PASP, 106, 983

Baraffe, I., Chabrier, G., Barman, T. S., Allard, F., \& Hauschildt, P. H. 2003, A\&A, 402, 701

Bochanski, J. J., West, A. A., Hawley, S. L., \& Covey, K. R. 2007, AJ, 133, 531

Burgasser, A. J., \& Kirkpatrick, J. D. 2006, ApJ, 645, 1485

Caballero, J. A. 2007, ApJ, 667, 520

Close, L. M., Zuckerman, B., Song, I., et al. 2007, ApJ, 660, 1492

Cruz, K. L., Kirkpatrick, J. D., \& Burgasser, A. J. 2009, AJ, 137, 3345

Epchtein, N., de Batz, B., Capoani, L., et al. 1997, The Messenger, 87, 27
Faherty, J. K., Riedel, A. R., Cruz, K. L., et al. 2016, ApJS, 225, 10

Gagné, J., Lafrenière, D., Doyon, R., Malo, L., \& Artigau, É. 2014, ApJ, 783, 121

Kirkpatrick, J. D., Cruz, K. L., Barman, T. S., et al. 2008, ApJ, 689, 1295

Kraus, A. L., \& Hillenbrand, L. A. 2009, ApJ, 703, 1511

Malo, L., Doyon, R., Lafrenière, D., et al. 2013, ApJ, 762, 88

Skrutskie, M. F., Cutri, R. M., Stiening, R., et al. 2006, AJ, 131, 1163

Zuckerman, B., Rhee, J. H., Song, I., \& Bessell, M. S. 2011, ApJ, 732, 61 